\title{
Visibilización de la mujer investigadora en espacios académicos de la Universidad Nacional
}

\author{
Ugalde Naranjo Jinette \\ Universidad Nacional de Costa Rica, Costa Rica \\ inette.ugalde.naranjo@una.cr \\ https://orcid.org/0000-0003-4176-1095 \\ Vasconcelos Vásquez Kattia Lizzett \\ Universidad Nacional de Costa Rica, Costa Rica \\ kvascon@una.cr \\ https://orcid.org/0000-0001-6803-4360 \\ Barquero Elizondo Ana Isabel \\ Universidad Nacional de Costa Rica, Costa Rica \\ ana.barquero.elizondo@una.cr \\ http://orcid.org/0000-0001-9670-128X \\ Valverde Chaves Jeannette \\ Universidad Nacional de Costa Rica, Costa Rica \\ jeannette.valverde.chaves@una.ac.cr \\ https://orcid.org/0000-0002-4695-2061 \\ Vega García Heidy \\ Universidad Nacional de Costa Rica, Costa Rica \\ heidy.vega.garcia@una.cr \\ https://orcid.org/0000-0002-2244-7634
}

\section{(ㅇ) $(\Theta \circ$}

La Revista Estudios es editada por la Universidad de Costa Rica y se distribuye bajo una Licencia Creative Commons Atribución-NoComercial-CompartirIgual 3.0 Costa Rica. Para más información envíe un mensaje a revistaestudios.eeg@ucr.ac.cr. 
Dossier: Mujeres y Educación en la Universidad Nacional

Red de Mujeres Investigadoras de la Universidad Nacional (UNA)

Montoya Hernández María Auxiliadora Universidad Nacional de Costa Rica, Costa Rica maria.montoya.hernandez@una.cr https://orcid.org/0000-0002-2371-3666

Gutiérrez Hernández Anayudel Universidad Nacional de Costa Rica, Costa Rica anayudel@una.ac.cr https://orcid.org/0000-0001-9924-9647

Recibido: 4 de agosto de 2020

Aceptado: 10 de noviembre de 2020

Resumen: El rol de las mujeres se ha minimizado en los diferentes ámbitos en los que se desempeñan, debido entre otras razones a las brechas de género. Según muestran estudios realizados a nivel nacional por la Universidad Nacional y la Universidad de Costa Rica, el crecimiento profesional de las mujeres académicas se ve obstaculizado por las cargas que la sociedad les asigna, especialmente aquellas orientadas al cuido de otras personas y del hogar.

Tomando en cuenta el contexto anterior, el objetivo de este artículo es exponer las acciones llevadas a cabo por la Red de Mujeres Investigadoras de la Universidad Nacional (MIRED) a la hora de generar espacios de reconocimiento, visibilización del trabajo y aportes de las académicas investigadoras de la Universidad Nacional.

Palabras claves: Costa Rica; equidad; género; interdisciplinaridad; vida Universitaria.

\section{Visibility of female researchers in academic spaces of the Universidad Nacional, Costa Rica}

\section{(C) $(0 \bigcirc$}

La Revista Estudios es editada por la Universidad de Costa Rica y se distribuye bajo una Licencia Creative Commons Atribución-NoComercial-CompartirIgual 3.0 Costa Rica. Para más información envíe un mensaje a revistaestudios.eeg@ucr.ac.cr. 
Dossier: Mujeres y Educación en la Universidad Nacional

Red de Mujeres Investigadoras de la Universidad Nacional (UNA)

\begin{abstract}
The role of women has been minimized in the different areas where they work, due to gender gaps. According to studies carried out at the national level by the Universidad Nacional and the Universidad de Costa Rica, the professional growth of academic women is hampered by the burdens that society assigns them, especially those aimed at caring for other people and the home.

Taking into account the previous context, the objective of this article is to "expose the actions carried out by the Red de Mujeres Investigadoras de la Universidad Nacional (MIRED) in the creation of spaces for recognition and visibility of the work and contributions of the academics- researchers from the Universidad Nacional.
\end{abstract}

Key Words: Costa Rica; equity; gender interdisciplinarity; university life.

\title{
Introducción
}

De acuerdo con su Estatuto Orgánico (UNA; 2015, 17) “La Universidad Nacional es una institución de educación superior pública con plena autonomía garantizada constitucionalmente. Tiene como misión histórica crear y transmitir conocimiento en favor del bienestar humano, mediante acciones que propician la transformación de la sociedad para llevarla a estadios superiores de convivencia. Honra la libertad, la diversidad, la búsqueda de la verdad y la sustentabilidad natural y cultural, en beneficio del conocimiento, la equidad, la justicia y la dignificación de la condición humana".

\section{(C) 100}

La Revista Estudios es editada por la Universidad de Costa Rica y se distribuye bajo una Licencia Creative Commons Atribución-NoComercial-CompartirIgual 3.0 Costa Rica. Para más información envíe un mensaje a 
Dossier: Mujeres y Educación en la Universidad Nacional

Red de Mujeres Investigadoras de la Universidad Nacional (UNA)

Uno de los valores que promulga esta casa de enseñanza es la equidad que promueve la igualdad de derechos y oportunidades sin ningún tipo de discriminación. Basado en este valor, en el 2010, se aprobó la Política para la Igualdad y Equidad de Género, la cual tiene como finalidad. "Propiciar una cultura universitaria libre de discriminación y marginación de género por medio de la incidencia en la organización y gestión de la Universidad Nacional así como su proyección externa, con el fin de contribuir con la justicia social y el desarrollo humano sostenible e Innovar la producción y la promoción del conocimiento mediante la incorporación de los aportes realizados desde los estudios de género para contribuir con la transformación de las condiciones que generen inequidad". (UNA, 2010, 12).

Años más tarde, en el 2016, se llevó a cabo un congreso universitario de investigación donde se planteó la necesidad de contar con espacios que visibilicen los aportes de las académicas investigadoras de la institución, hecho que motivó en el 2017, la creación de la Red de Mujeres Investigadoras de la Universidad Nacional, en adelante MIRED, como una iniciativa que impulsa la investigación, formación y capacitación de las académicas y estudiantes de la institución al basarse en los principios de igualdad, inclusión, solidaridad y cooperación. De esta forma, MIRED fortalece lo propuesto en las políticas institucionales mencionadas. MIRED es una red temática conformada por un grupo inter y multidisciplinario de académicas que lideran y participan en programas, proyectos y actividades académicas (PPA) desde sus instancias. Ejecuta diversas acciones para la 
Dossier: Mujeres y Educación en la Universidad Nacional

Red de Mujeres Investigadoras de la Universidad Nacional (UNA)

transferencia y construcción de conocimientos y aspira a incidir en la acción sustantiva de investigación, extensión, docencia y producción de las mujeres universitarias, en concordancia con los principios, valores y fines de la Universidad Nacional.

Desde sus orígenes, MIRED se ha propuesto apoyar la construcción de espacios de intercambio y reconocimiento del trabajo realizado por mujeres académicas investigadoras de la institución, de modo que sean visibilizados los esfuerzos y alcances obtenidos por estas funcionarias en el ámbito universitario, nacional e internacional.

Las instancias fundadoras de la MIRED son las siguientes:

a) Vicerrectoría de Investigación

b) Programa de Gestión de Bosques (PGB) del Instituto de Investigación y Servicios Forestales (INISEFOR)

c) Programa de Atención Integral de la Persona Adulta Mayor (PAIPAM) del Centro de Estudios Generales

d) Escuela Ecuménica de Ciencias de la Religión

e) Programa Desarrollo de Habilidades Blandas Empresariales (PROHABLA) de la Escuela de Administración

f) Programa de Comercio y Negocios Internacionales de la Escuela de Relaciones Internacionales

g) Laboratorio de Materiales Industriales LAMI del Departamento de Física

\section{(C) $(0 \odot$}

La Revista Estudios es editada por la Universidad de Costa Rica y se distribuye bajo una Licencia Creative Commons Atribución-NoComercial-CompartirIgual 3.0 Costa Rica. Para más información envíe un mensaje a revistaestudios.eeg@ucr.ac.cr. 
Dossier: Mujeres y Educación en la Universidad Nacional

Red de Mujeres Investigadoras de la Universidad Nacional (UNA)

MIRED cuenta con una comisión encargada de diseñar, ejecutar diversas actividades tales como cursos cortos, seminarios, congresos, charlas, elaborar proyectos y otras actividades de difusión del trabajo de las académicas; esta comisión está integrada por académicas de la Escuela de Administración, Instituto de Investigación y Servicios Forestales (INISEFOR), Escuela de Relaciones Internacionales, Centro de Estudios Generales, Escuela Ecuménica de Ciencias de la Religión y de la Vicerrectoría de Investigación.

\section{Referentes teóricos}

La igualdad de género va más allá de roles asignados por la sociedad, se trata de tener igualdad de condiciones a la hora de lograr oportunidades sociales, laborales, familiares y económicas. Según la Organización de Naciones Unidas (2020) la desigualdad de género es un fenómeno generalizado en el que las mujeres carecen de acceso a empleo decente y enfrentan diferencias salariales por motivo de género. En todo el mundo las mujeres y las niñas son privadas sistemáticamente de acceso a la educación y a la atención de la salud, están subrepresentadas en la adopción de decisiones económicas y políticas y son víctimas de la violencia y discriminación. De ahí que la igualdad entre los géneros no es sólo un derecho humano básico, sino que su logro tiene enormes ramificaciones socioeconómicas y por ello fortalecer a las mujeres da un impulso a las economías florecientes, a la productividad y al crecimiento.

\section{(C) $(0 \odot$}

La Revista Estudios es editada por la Universidad de Costa Rica y se distribuye bajo una Licencia Creative Commons Atribución-NoComercial-CompartirIgual 3.0 Costa Rica. Para más información envíe un mensaje a 
Dossier: Mujeres y Educación en la Universidad Nacional

Red de Mujeres Investigadoras de la Universidad Nacional (UNA)

Por su parte, Villoro citado en el documento Brechas de Género de la CEPAL, explica sobre los retos pendientes para garantizar el acceso a la salud sexual y reproductiva para cerrar las brechas de género, indica que la desigualdad de las mujeres:

...constituye un caso especial entre todas las discriminaciones sociales. En efecto, a diferencia de otros casos, no constituyen una clase social, ni un grupo específico; no son una comunidad, ni una minoría social o racial, atraviesan todos los grupos y pueblos y, en todos ellos, son una inseparable mitad. Acabar con las condiciones que han permitido su desigualdad social y política sería, después de la liberación de los esclavos, la mayor revolución emancipadora (Villoro, 1997, citado en CEPAL, 2010, p. 2).

Según el artículo de Aguilar publicado en el Programa Estado de la Nación:

Las mujeres en Costa Rica, que representan la mitad de toda la población, son más educadas que los hombres, pues constituyen el $57 \%$ de las personas con títulos universitarios. Sin embargo, el mejor perfil educativo no se traduce en mayores oportunidades para insertarse en el mercado laboral. (Aguilar, 2020, párr. 3)

En la Encuesta Nacional de Uso del Tiempo del 2017, de ahora en adelante ENUT, se alude al planteamiento de la Organización Internacional del Trabajo (OIT) la cual expone que: "la inequidad en la distribución y uso del tiempo entre mujeres y hombres es un determinante importante de las desigualdades en el trabajo y en el empleo" (INEC, 2017, p. 5). En este sentido, la OIT reconoce que "al ser el trabajo doméstico no remunerado y de cuidados una barrera para el pleno desarrollo de las mujeres, éste debe reconocerse como un asunto público que compete a los

Estados, gobiernos locales, organizaciones, empresas y familias" (INEC, 2017, p.



La Revista Estudios es editada por la Universidad de Costa Rica y se distribuye bajo una Licencia Creative Commons Atribución-NoComercial-CompartirIgual 3.0 Costa Rica. Para más información envíe un mensaje a revistaestudios.eeg@ucr.ac.cr. 
Dossier: Mujeres y Educación en la Universidad Nacional

Red de Mujeres Investigadoras de la Universidad Nacional (UNA)

$5)$.

Según los resultados de la ENUT, se encuentra que, en Costa Rica, las mujeres ocupan 35:49 horas semanales para realizar el trabajo doméstico no remunerado, mientras que los hombres ocupan 13:42 horas semanales. Como parte de esta encuesta se obtiene que la carga global de trabajo de las mujeres, es decir, el trabajo remunerado y no remunerado, es de 69 horas y 53 minutos mientras que el de los hombres es de 62 horas y 59 minutos dejando en evidencia que las mujeres tienen un recargo de trabajo de más de siete horas (INEC, 2017, p. 54). En el contexto anterior, el estudio realizado por Meza muestra que un factor incidente en el menor avance de las mujeres en el Régimen Académico de la Universidad de Costa Rica puede estar relacionado con "la división sexual del trabajo, que determina la carga que llevan las mujeres de trabajo doméstico no remunerado, en especial, el tiempo que consume la crianza de sus hijos e hijas". (2019, p. 4) Según el estudio mencionado en líneas anteriores, al año 2006 hubo un aumento de la participación de las mujeres en los estudios realizados por la oficina de Carrera Académica de la UNA y un incremento en asignación en todas las categorías académicas que asigna esta Oficina, que a saber son profesora I, profesora II, profesora II con incentivo y catedrática. Sin embargo, la categoría más alta que corresponde a catedráticas continua con baja participación femenina y a esa fecha (2016) el $66.1 \%$ de las personas catedráticas de la UNA correspondía a hombres y solamente un 33,9\% a mujeres. (Meza, 2019, p. 19). Meza menciona además que la perspectiva de algunas mujeres académicas de esta casa de enseñanza es que

\section{(C) $(0 \odot$}

La Revista Estudios es editada por la Universidad de Costa Rica y se distribuye bajo una Licencia Creative Commons Atribución-NoComercial-CompartirIgual 3.0 Costa Rica. Para más información envíe un mensaje a 
Dossier: Mujeres y Educación en la Universidad Nacional

Red de Mujeres Investigadoras de la Universidad Nacional (UNA)

sus compañeros de trabajo ingresan a laborar a la universidad al mismo tiempo que ellas, pero han avanzado más rápido en carrera académica. (Meza, 2019, p.23).

Por su parte, Fernández (2014) muestra la existencia de diferencias de género en el número de solicitudes de ascenso en Régimen de Carrera Académica en la Universidad Nacional y que las mayores brechas se encuentran en las categorías más altas. Por ejemplo, en la categoría de Profesora II, las mujeres representan un $39 \%$ de las solicitudes procesadas, mientras que los hombres un $61 \%$. Para la categoría de Catedrático, las mujeres un $29 \%$ de las solicitudes y los hombres un $71 \%$. En el estudio de Fernández se evidencian datos suministrados por el Área de

Desarrollo Organizacional de Recursos Humanos, en los cuales se muestra que:

...a diciembre del 2010, había un total de 638 personas académicas propietarias (266 mujeres y 372 hombres) de las cuales 252 se encontraban rezagadas (111 mujeres y 141 hombres) representando el rezago de las mujeres un $43 \%$ y el de los hombres un 38\% (Fernández, 2014, p.4).

En el contexto internacional Krefting (2003) citada por Meza, (2019 p 3), menciona que:

en Estados Unidos, las mujeres han alcanzado paridad con los hombres en acceso a la educación en todos los niveles, excepto el Doctorado, que constituye un requisito indispensable para trabajar en la academia. Al mismo tiempo, encuentra que un porcentaje alto de mujeres abandonan los estudios doctorales sin obtener su grado. Otro hallazgo fue que el porcentaje de docentes en propiedad en las universidades es del 33\%, lo que significa un porcentaje menor que el de las que cuentan con doctorados.

\section{(ब) $\odot \odot$}

La Revista Estudios es editada por la Universidad de Costa Rica y se distribuye bajo una Licencia Creative Commons Atribución-NoComercial-CompartirIgual 3.0 Costa Rica. Para más información envíe un mensaje a revistaestudios.eeg@ucr.ac.cr. 
Dossier: Mujeres y Educación en la Universidad Nacional

Red de Mujeres Investigadoras de la Universidad Nacional (UNA)

Como se evidencia en los diferentes estudios, las mujeres académicas se encuentran en desigualdad de condiciones para crecer profesionalmente, en algunos casos esto se debe al exceso de trabajo no remunerado y remunerado que les impide la producción. Adicionalmente, los roles de crianza y cuido asignados socialmente a las mujeres, afectan la carga de tiempo y las posibilidades de aumentar su producción intelectual.

\section{Experiencias desarrolladas por la Red de Mujeres Investigadoras de la Universidad Nacional}

En la era de la comunicación, la MIRED ha establecido líneas de interacción menos convencionales para ser vocera de las mujeres académicas que de una u otra manera enfrentan una realidad estructural, social e histórica que en muchas ocasiones dificulta la obtención de un mejor posicionamiento en el escalafón universitario. Durante el periodo 2017-2020, se realizaron diversas actividades que abordan temáticas de interés para las mujeres académicas, entre ellas se encuentran:

- $\quad$ Actividades para el desarrollo de habilidades en la escritura científica.

- Uso de herramientas novedosas para la investigación y la docencia.

- Capacitación sobre la utilización de nuevos sistemas para el ingreso y registro de producción intelectual al Régimen de Carrera Académica.

\section{(C) $(0 \odot$}

La Revista Estudios es editada por la Universidad de Costa Rica y se distribuye bajo una Licencia Creative Commons Atribución-NoComercial-CompartirIgual 3.0 Costa Rica. Para más información envíe un mensaje a 
Dossier: Mujeres y Educación en la Universidad Nacional

Red de Mujeres Investigadoras de la Universidad Nacional (UNA)

Promoción y motivación para el intercambio con expertas en áreas como derechos humanos, igualdad de género, migraciones, entre otras.

- Realización de talleres, charlas y eventos con especialistas en diferentes temas.

La internacionalización es un proyecto importante dentro del quehacer de la MIRED, el cual se desarrolla mediante el fortalecimiento y la creación de nexos con académicas investigadoras de diversas universidades, tanto a nivel latinoamericano, como de Europa, Canadá y Estados Unidos. Algunas de las actividades realizadas desde el 2017 incluyeron la venida a la Universidad Nacional de pares de España, Brasil y México, quienes participaron en los Seminarios y cursos ofrecidos por MIRED.

A manera de ejemplo, algunas de las actividades más relevantes ejecutadas por MIRED en el periodo 2017- 2018 fueron la realización de encuentros de académicas en el marco del Día Internacional de la Mujer. Estos encuentros permitieron generar un espacio para el reconocimiento de académicas que a lo largo de su trayectoria en la UNA han sobresalido por sus trabajos de investigación y que, a su vez, han sido reconocidas y citadas por otros expertos, así como también dentro de las diferentes facultades universitarias.

En torno a lo anterior, en el 2017, se contó con la participación de la PhD. María Lourdes Bernart, de la Universidad Federal de Pato Branco, Brasil, quien llevó a

La Revista Estudios es editada por la Universidad de Costa Rica y se distribuye bajo una Licencia Creative Commons Atribución-NoComercial-CompartirIgual 3.0 Costa Rica. Para más información envíe un mensaje a revistaestudios.eeg@ucr.ac.cr. 
Dossier: Mujeres y Educación en la Universidad Nacional

Red de Mujeres Investigadoras de la Universidad Nacional (UNA)

cabo un conversatorio sobre metodologías de la investigación en el contexto académico. Asimismo, durante la celebración del Mes de la Ciencia, en agosto del 2018 se contó con la visita de la Dra. Raquel Guereca, integrante de la Red Mexicana de Ciencia, Tecnología y Género, quien impartió varios talleres sobre igualdad de género y currículum oculto.

\section{Seminarios internacionales organizados por la MIRED}

Durante el 2019 y el 2020 se realizaron seminarios internacionales en los cuales se contó con gran participación de académicas universitarias, lo que permitió evidenciar el trabajo que realizan desde las diferentes áreas del quehacer universitario. Estos eventos también son vistos como una oportunidad de crecimiento profesional, pues se gestionan alianzas para la publicación de los trabajos presentados, lo que a la vez da valor y reconocimiento institucional al aporte que realizan las académicas. Los seminarios se transforman en un espacio de intercambio de experiencias con otras mujeres académicas e investigadoras de diferentes universidades nacionales e internacionales, lo que impulsa la generación de alianzas para el trabajo colaborativo internacional. Contar con el conocimiento y experiencia del quehacer de diferentes mujeres en sus países de origen amplía la visión para el mejoramiento de estrategias y objetivos que permiten fortalecer la participación de las académicas en la vida universitaria.

\section{(c) (i) (2) (2)}

La Revista Estudios es editada por la Universidad de Costa Rica y se distribuye bajo una Licencia Creative Commons Atribución-NoComercial-CompartirIgual 3.0 Costa Rica. Para más información envíe un mensaje a 
Dossier: Mujeres y Educación en la Universidad Nacional

Red de Mujeres Investigadoras de la Universidad Nacional (UNA)

\section{Datos relevantes de los Seminarios Internacionales}

I Seminario Internacional de la Red de Mujeres Investigadoras de la Universidad Nacional. Marzo de 2019.

En este seminario se registró la participación de 90 personas académicas, estudiantes y administrativas, de las cuales 26 presentaron ponencias sobre los siguientes temas:

- Madres-estudiantes universitarias en el Campus Liberia de la Universidad Nacional de Costa Rica.

- La experiencia de las mujeres docentes en la robótica educativa en el marco de un proyecto de extensión universitaria.

- Manejo de polen de especies forestales como herramienta para el mejoramiento genético.

- Tripanosomiasis canina: una zoonosis presente en la comunidad de Getsemaní de San Rafel de Heredia.

- ¿A más pluralismo religioso mayor igualdad de género?

- Identificando percepciones y manifestaciones de discriminación en la Universidad Nacional: Hacia un ambiente universitario libre de toda forma de discriminación.

- Implicaciones de la condición de interinazgo en la UNA: Caracterización del perfil de población interina de la Facultad de Ciencias Sociales y la Facultad de Filosofía y Letras, 2018.

\section{(C) $\odot \odot \odot$}

La Revista Estudios es editada por la Universidad de Costa Rica y se distribuye bajo una Licencia Creative Commons Atribución-NoComercial-CompartirIgual 3.0 Costa Rica. Para más información envíe un mensaje a revistaestudios.eeg@ucr.ac.cr. 
Dossier: Mujeres y Educación en la Universidad Nacional

Red de Mujeres Investigadoras de la Universidad Nacional (UNA)

Impacto de los desastres Otto y Nate en mujeres del campo, recrudecimiento de la violencia.

- Asociación de mujeres organizadas de Biolley (ASOMOBI). Un ejemplo de emprendedurismo y liderazgo en turismo rural comunitario en el distrito de Biolley, cantón Buenos Aires.

- Vinculación de las mujeres indígenas maleku con el hogar y sus transformaciones a partir de la elaboración y comercialización de artesanía.

- La mujer rural emprendedora ante el cambio climático en Guanacaste.

- $\quad$ Cultivo in vitro de Tectona grandis: biotecnología en el sector forestal.

- Posibilidades de aprovechamiento y almacenamiento de carbono en rodales de Guadua angustifolia (bambú), Península de Osa, Costa Rica.

- Distribución geográfica de Paragonimus mexicanus y Paragonimus caliensis en cangrejos de agua dulce de Costa Rica.

- Avances y desafíos para alcanzar la igualdad y equidad de género en la Universidad Nacional.

- De furias infernales a cristianas devotas: las mujeres indígenas en la voz del misionero jesuita Antonio Sepp (1655-1733).

- $\quad$ Ciudadanía y contrato sexual: análisis de las barreras que las mujeres enfrentan para el acceso a la anticoncepción oral de emergencia en Costa Rica.

- Barreras en la educación superior.

- Políticas de género que afectan o no la formalización de créditos bancarios

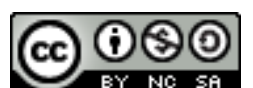

La Revista Estudios es editada por la Universidad de Costa Rica y se distribuye bajo una Licencia Creative Commons Atribución-NoComercial-CompartirIgual 3.0 Costa Rica. Para más información envíe un mensaje a revistaestudios.eeg@ucr.ac.cr. 
Dossier: Mujeres y Educación en la Universidad Nacional

Red de Mujeres Investigadoras de la Universidad Nacional (UNA)

Incorporación de género en la Facultad de Ciencias Sociales de la Universidad Nacional

- $\quad$ Características de la publicación de académicas de la Universidad Nacional de Costa Rica en revistas institucionales.

- $\quad$ La violencia contra la mujer en los medios de comunicación.

Para esta actividad la invitada internacional fue la PhD. Beatriz Urraca, Especialista en Humanidades y Género de Widener University, Pennsylvania, Filadelfia, Estados Unidos. Brindó la conferencia Mujeres en la academia: Desafíos estructurales y políticas de identidad. Asimismo, participa la Máster Chelsy Abba, Widener University, Pennsylvania, Filadelfia, Estados Unidos, quien expone su experiencia de estudios doctorales en comunidades de Costa Rica y Nicaragua.

Como parte del reconocimiento a las académicas investigadoras de la Universidad Nacional se invita a la Dra. Silvia Rojas Herrera, coordinadora del Programa Comunitario Rural Costero, quien brinda la conferencia Más allá de las significaciones asignadas al mar. Una visión interdiciplinaria de la investigación feminista.

\section{(C) $(00$}

La Revista Estudios es editada por la Universidad de Costa Rica y se distribuye bajo una Licencia Creative Commons Atribución-NoComercial-CompartirIgual 3.0 Costa Rica. Para más información envíe un mensaje a revistaestudios.eeg@ucr.ac.cr. 
Dossier: Mujeres y Educación en la Universidad Nacional

Red de Mujeres Investigadoras de la Universidad Nacional (UNA)

Il Seminario de la Red de Mujeres Investigadoras de la Universidad Nacional: Mujer, Derechos Humanos y Migración. Marzo de 2020.

En esta ocasión participan aproximadamente 70 personas entre académicas, estudiantes y administrativas, pertenecientes a las facultades de Ciencias Sociales, Filosofía y Letras, Ciencias Exactas y Naturales, Ciencias de la Tierra y el Mar, así como de del Centro de Estudios Generales, Centro de Investigación y Docencia en Educación, Centro de Investigación, Docencia y Extensión Artística, así como de la Oficina de Comunicación, la Vicerrectoría de Docencia, Biblioteca Joaquín García Monge y Vicerrectoría de Extensión. Además, en este evento participan como ponentes estudiantes del Doctorado en Mediación Pedagógica de la Universidad La Salle, una de ellas desde Colombia. También participa una estudiante del Doctorado de Estudios Latinoamericanos de la Universidad Nacional quien desde España hace la disertación de su ponencia.

Los temas abordados por las académicas, estudiantes y administrativas en este seminario son los siguientes:

- $\quad$ El color de la violencia. Programa de prevención de la violencia machista desde las aulas.

- Acceso educativo y laboral de las mujeres en el contexto de las STEM: una revisión quinquenal de las estadísticas estudiantiles del Departamento de Registro de la Universidad Nacional (UNA).

\section{(C) $(0 \odot$}

La Revista Estudios es editada por la Universidad de Costa Rica y se distribuye bajo una Licencia Creative Commons Atribución-NoComercial-CompartirIgual 3.0 Costa Rica. Para más información envíe un mensaje a revistaestudios.eeg@ucr.ac.cr. 
Dossier: Mujeres y Educación en la Universidad Nacional

Red de Mujeres Investigadoras de la Universidad Nacional (UNA)

"Yo hubiera querido tener menos hijos": Reflexiones de mujeres migrantes en torno a sus derechos sexuales y reproductivos.

- La trata en los procesos migratorios: una manifestación de violencia contra las mujeres en América Latina.

- $\quad$ Ser mujer docente. Significados y sentidos de estudiantes de licenciatura en ciencias sociales en el desarrollo de su práctica pedagógica.

- ¿Cómo la robótica educativa impacta en las mujeres estudiantes de secundaria? Un análisis realizado con Python

- $\quad$ El rol de la mujer en el desarrollo comunitario, caso Ugrosanta, Santa Cruz de Turrialba, Costa Rica.

- Mujeres en condición de interinazgo, la lucha por la estabilidad laboral y el crecimiento profesional en la UNA, 2020.

- Mujeres migrantes centroamericanas y el reto de la violencia de género.

- La mujer rural emprendedora ante el cambio climático en Guanacaste.

- Y la mujer votó: Retos político-electorales de las mujeres a 70 años del voto femenino en Costa Rica.

- $\quad$ Experiencia de la implementación de políticas de género en la Facultad de Ciencias Sociales de la Universidad Nacional.

- $\quad$ Aportes y reflexiones del acompañamiento desde las artes y la educación somática a la salud.

- $\quad$ La trayectoria histórica de la anticoncepción en Costa Rica.

- Del centralismo en el discurso de la sobrepoblación hasta su transformación en un derecho humano.

\section{(c) (i) (9) (2)}

La Revista Estudios es editada por la Universidad de Costa Rica y se distribuye bajo una Licencia Creative Commons Atribución-NoComercial-CompartirIgual 3.0 Costa Rica. Para más información envíe un mensaje a revistaestudios.eeg@ucr.ac.cr. 
Dossier: Mujeres y Educación en la Universidad Nacional

Red de Mujeres Investigadoras de la Universidad Nacional (UNA)

En este segundo seminario participan como invitadas internacionales la PhD. María Lourdes Bernart, profesora asociada de la Universidad Tecnológica Federal de Paraná UTFPR, Campus Pato Branco, Brasil, especialista en políticas públicas de educación para población rural, políticas públicas migratorias, políticas públicas para personas adultas mayores, ciudades y comunidades amigables con las personas adultas mayores, educación en derechos humanos y diversidades. La Dra. Bernart ofreció la conferencia inaugural sobre Género y Migración: una mirada a las mujeres migrantes. También la Máster Rosario Pedraza Salazar, presidenta de la Costa Rica Chamber of Commerce con sede en Miami, Estados Unidos, quien impartió la charla virtual Creciendo como persona fuera de Costa Rica.

Además, participó la Dra. Rocío Loría Bolaños del Instituto Regional de Estudios en Sustancias Tóxicas (IRET) de la Universidad Nacional, coordinadora de la Maestría en Bioética, quien ofreció la charla Desafíos en el abordaje del género y la etnicidad en la investigación: experiencia de estudios de violencia contra mujeres indígenas.

\section{Otras actividades que se han generado desde la MIRED:}

En cumplimiento de sus fines y propósitos MIRED, ha elaborado un conjunto diverso de actividades entre las que se destacan las siguientes:

- La elaboración de un instrumento para el Diagnóstico de Necesidades e Intereses de la Red.

\section{(C) $(\Theta \odot$}

La Revista Estudios es editada por la Universidad de Costa Rica y se distribuye bajo una Licencia Creative Commons Atribución-NoComercial-CompartirIgual 3.0 Costa Rica. Para más información envíe un mensaje a 
Barquero-Elizondo Ana Isabel Valverde Chaves Jeannette

Vega-García Heidy

Montoya-Hernández María Auxiliadora

Gutiérrez-Hernández Anayudel

Dossier: Mujeres y Educación en la Universidad Nacional

Red de Mujeres Investigadoras de la Universidad Nacional (UNA)

La celebración del Día Internacional de la Mujer 2017 en la fecha del 08 de marzo del 2017, en esta actividad se entregó por primera vez el premio a la mujer investigadora.

- La presentación del libro titulado Las competencias docentes en la educación superior escrito por la Dra. Carolina España Chavarría de la Escuela de Secretariado Profesional de la Universidad Nacional. La actividad se llevó a cabo el 1 de septiembre de 2017.

- La ejecución del taller: Experiencias y recomendaciones para lograr publicaciones científicas exitosas, impartido por Suyen Alonso, destacada editora e investigadora de la Universidad Nacional.

- El curso de capacitación de la herramienta WEB 2.0 durante los meses de enero y febrero de 2018, impartido por la Vicerrectoría de Docencia.

- $\quad$ El Taller de Capacitación para presentación de producción intelectual ante carrera académica. La actividad se llevó a cabo el 12 de abril de 2018.

- La participación en el programa UNA Mirada con el tema Mujeres en la Ciencia en el marco del Día Internacional de la Mujer en marzo 2019.

- $\quad$ En el 2019 se publicó el primer libro de la Red de Mujeres Investigadoras, el cual contiene un compendio de aproximadamente quince artículos presentados en el I Seminario de la Red de Mujeres Investigadoras de la UNA.

- $\quad$ Se realizan gestiones con la Revista Estudios UCR, para la publicación de un dossier producto del II Seminario Internacional de la Red de Mujeres

\section{(C) $(\Theta \odot$}

La Revista Estudios es editada por la Universidad de Costa Rica y se distribuye bajo una Licencia Creative Commons Atribución-NoComercial-CompartirIgual 3.0 Costa Rica. Para más información envíe un mensaje a 
Dossier: Mujeres y Educación en la Universidad Nacional

Red de Mujeres Investigadoras de la Universidad Nacional (UNA)

Investigadoras de la UNA. También, se insta a las académicas a presentar anualmente sus artículos a esta revista.

- $\quad$ Participación de la Máster Lorna Peraza, directora y Fundadora de Genius Lab con la charla Entrando al mundo de la gamificación.

- $\quad$ En el 2020 da inicio una investigación sobre el impacto del teletrabajo como medida ante el COVID-19.

- Participación en la charla virtual Retos y Oportunidades del Teletrabajo a partir del COVID-19 con el tema Retos desde la academia para la gestión del teletrabajo en la acción sustantiva de la Universidad Nacional.

- Participación en el taller Emprendimiento en el Aula, en el cual se gestiona con representantes de las cinco universidades públicas un proyecto que inste a las académicas investigadoras a emprender.

\section{¿Quiénes forman parte de la Red de Mujeres Investigadoras?}

Se consideran miembros de la MIRED todas aquellas académicas que estén interesadas en evidenciar el trabajo que generan desde sus proyectos o unidades y en potenciar su crecimiento profesional. Hasta el 2020 se cuenta con una base de datos de 487 académicas que pertenecen a las diferentes sedes y facultades universitarias. La incorporación de nuevos miembros de la red se propone a través de invitaciones formales por medios electrónicos, así como en las diferentes actividades que realice la MIRED.

\section{(ब) $\odot \odot$}

La Revista Estudios es editada por la Universidad de Costa Rica y se distribuye bajo una Licencia Creative Commons Atribución-NoComercial-CompartirIgual 3.0 Costa Rica. Para más información envíe un mensaje a 
Dossier: Mujeres y Educación en la Universidad Nacional

Red de Mujeres Investigadoras de la Universidad Nacional (UNA)

\section{Participación estudiantil}

Por el tipo de actividades que propone la MIRED, también se cuenta con la colaboración de varios grupos de estudiantes, sobre todo de la Escuela de Administración, de la Escuela de Relaciones Internacionales, estudiantes vinculados con la Vicerrectoría de Investigación y con el Programa de Atención Integral de la Persona Adulta Mayor (PAIPAM), quienes desempeñan diversas funciones, tales como asistentes en las mesas de trabajo, edecanes, manejo de comunicación y redes sociales, aspectos logísticos, entre otros.

Además, para el II Seminario Internacional de la MIRED, se contó con la participación de estudiantes ponentes provenientes de la Escuela de Relaciones Internacionales y de la Escuela de Arte y Comunicación Visual y de la Escuela de Arte Escénico.

\section{Vínculos con actores sociales nacionales e internacionales:}

Para la generación de vínculos con los diferentes actores se establecen relaciones colaborativas con centros de excelencia, nacionales o internacionales, instituciones gubernamentales, otras redes a nivel internacional, ONG, entre otros. Esto se logra a través de la representación académica en diferentes actividades o instancias, por ejemplo, la participación en ONU Mujer, Asociación de Mujeres Betas, participación en la comisión de política de género del MICITT, entre otras. De igual manera, en

\section{(c) (i) (9) (2)}

La Revista Estudios es editada por la Universidad de Costa Rica y se distribuye bajo una Licencia Creative Commons Atribución-NoComercial-CompartirIgual 3.0 Costa Rica. Para más información envíe un mensaje a 
Dossier: Mujeres y Educación en la Universidad Nacional

Red de Mujeres Investigadoras de la Universidad Nacional (UNA)

el ámbito nacional, esta red busca estrechar vínculos con instituciones y organizaciones gubernamentales que trabajan temas relacionados con las mujeres y la investigación como por ejemplo el Ministerio de Educación Pública, el Ministerio de Ciencia y Tecnología, la Academia Nacional de Ciencias, el Instituto Nacional de las Mujeres (INAMU), otras universidades como Universidad de Costa Rica (UCR), Universidad Estatal a Distancia (UNED), Universidad Técnica Nacional (UTN), Instituto Tecnológico (TEC); organizaciones no gubernamentales, municipalidades, entre otros.

En el ámbito internacional la MIRED busca estrechar lazos y establecer contacto, por medio de intercambios con organismos internacionales como UNESCO, ONU Mujeres, redes de investigación de instituciones de educación superior, institutos de investigación, entre otros.

\section{Propuestas estratégicas para la consolidación de la MIRED}

Como parte de la consolidación actual de la Red y para su crecimiento a corto y mediano plazo, se plantea la necesidad de promover la divulgación del quehacer académico llevado a cabo por las integrantes de la Red. Se espera lograr esto por medio de canales de comunicación variados, por ejemplo, reseñas de la MIRED en el Periódico Campus, participación en programas como UNA Mirada, programas de radio y televisión, publicación de artículos de opinión en páginas web, periódicos

\section{(c) (i) (2)}

La Revista Estudios es editada por la Universidad de Costa Rica y se distribuye bajo una Licencia Creative Commons Atribución-NoComercial-CompartirIgual 3.0 Costa Rica. Para más información envíe un mensaje a 
Dossier: Mujeres y Educación en la Universidad Nacional

Red de Mujeres Investigadoras de la Universidad Nacional (UNA)

nacionales, publicaciones en redes sociales como el Facebook de las Unidades Académicas participantes, entre otros.

También se plantea la necesidad de contar con un boletín digital informativo de la red, el cual será distribuido a todos los aliados estratégicos para fomentar la producción intelectual de las mujeres investigadoras por medio de actividades tales como presentaciones de libros, ponencias en eventos nacionales e internacionales, entre otros

Finalmente es oportuno mencionar que, en el ejercicio de búsqueda de su consolidación, la Red de Mujeres visualiza la realización de proyectos orientados al diseño y ejecución de investigación y extensión en temas vinculados con lo planteado en la Políticas de igualdad y equidad de género de la UNA, y a la promoción y difusión de actividades que favorezcan el desarrollo de liderazgos de las mujeres universitarias.

\section{Referencias Bibliográficas}

Aguilar, M. (7 de marzo 2020). Queda mucho camino por recorrer... en la equidad de género. Recuperado de: https://estadonacion.or.cr/queda-mucho-caminoporrecorrer-en-la-equidad-de-genero/

\section{(C) $(0 \odot$}

La Revista Estudios es editada por la Universidad de Costa Rica y se distribuye bajo una Licencia Creative Commons Atribución-NoComercial-CompartirIgual 3.0 Costa Rica. Para más información envíe un mensaje a revistaestudios.eeg@ucr.ac.cr. 
Dossier: Mujeres y Educación en la Universidad Nacional

Red de Mujeres Investigadoras de la Universidad Nacional (UNA)

Cepal. (2018). Brechas de género: Retos pendientes para garantizar el acceso a la salud sexual y reproductiva, y para cerrar las brechas de género. Disponible en: https://crpd.cepal.org/3/sites/crpd3/files/presentations/panel2_marcelaeternod.pdf

Elizondo, A.; Novo, A. y Silvestre, M. (2010). Igualdad de mujeres y hombres en las universidades españolas. Instituto de la Mujer. Madrid: España.

Fernández, D. (2014). Condiciones que limitan el ascenso a carrera académica del personal académico propietario de la Universidad Nacional. Informe final de Investigación. Recuperado de: http://www.iem.una.ac.cr/index.php/publicaciones/serieinvestigacionesiem/condiciones

Instituto Nacional de Estadística y Censos de Costa Rica. (2017). Encuesta Nacional de Uso del Tiempo. Recuperado de:

https://www.inec.cr/sites/default/files/documetos-biblioteca-virtual/reenut2017.pdf

Meza, S. (2019). Carreras académicas de mujeres en la Universidad de Costa Rica: Un reto para la igualdad. Revista Electrónica Actualidades Investigativas en Educación 19(1), 1-32. DOI:10.15517/aie. v19i1.35716. Recuperado de:

https://www.scielo.sa.cr/pdf/aie/v19n1/1409-4703-aie-19-01-314.pdf

\section{(C) $(0 \odot$}

La Revista Estudios es editada por la Universidad de Costa Rica y se distribuye bajo una Licencia Creative Commons Atribución-NoComercial-CompartirIgual 3.0 Costa Rica. Para más información envíe un mensaje a revistaestudios.eeg@ucr.ac.cr. 
Dossier: Mujeres y Educación en la Universidad Nacional

Red de Mujeres Investigadoras de la Universidad Nacional (UNA)

ONU Mujeres. (2020) ONU-Mujeres: Entidad de las Naciones Unidas para la Igualdad de Género y el Empoderamiento de las Mujeres. Recuperado de: https://www.un.org/es/sections/issues-depth/gender-equality/index.html

Universidad Nacional (2015). Estatuto Orgánico. Recuperado de: https://www.documentos.una.ac.cr

Universidad Nacional (2016). Política para la igualdad y equidad de género en la Universidad Nacional y Plan de acción /. Instituto de Estudios de la Mujer, Universidad Nacional. Heredia, Costa Rica

La Revista Estudios es editada por la Universidad de Costa Rica y se distribuye bajo una Licencia Creative Commons Atribución-NoComercial-CompartirIgual 3.0 Costa Rica. Para más información envíe un mensaje a revistaestudios.eeg@ucr.ac.cr. 